\title{
Spectrally Efficient Waveforms for the Return Link in Satellite Communication Systems
}

\author{
Svilen Dimitrov*, Niccoló Privitera ${ }^{\dagger}$, Rosalba Suffritti ${ }^{\dagger}$, Gabriele Boccolini ${ }^{\ddagger}$, Adegbenga B. Awoseyila ${ }^{\S}$ \\ and Barry G. Evans ${ }^{\S}$ \\ *German Aerospace Center (DLR), Satellite Networks Department, 82234 Wessling, Germany, e-mail: svilen.dimitrov@dlr.de \\ ${ }^{\dagger}$ Mavigex S.r.l., ICT Solutions, 40125 Bologna, Italy, e-mail: \{niccolo.privitera,rsuffritti\}@mavigex.com \\ $\ddagger$ Galician R\&D Center in Advanced Telecommunications (GRADIANT), 36310 Vigo, Spain, e-mail: gboccolini@gradiant.org \\ ${ }^{\S}$ University of Surrey, Centre for Communication Systems Research, GU27XH, UK, e-mail: \{a.awoseyila, b.evans\}@ surrey.ac.uk
}

\begin{abstract}
In this paper, we study the applicability of terrestrial mobile waveforms in the return link of a high throughput satellite (HTS) communication system. These include orthogonal frequency division multiple access (OFDMA), single-carrier frequency division multiple access (SC-FDMA) and filter bank multi-carrier (FBMC). Key solutions to the challenges in a geostationary orbit (GEO) satellite channel, such as synchronization and non-linear distortion, are presented. A globalpositioning-system-(GPS)-based approach for synchronization acquisition is proposed, while suitable algorithms are studied for timing/frequency offset estimation and synchronization tracking. The spectral and power efficiencies of the schemes are optimized by means of an intermodulation interference (IMI) cancelling receiver, and these are compared to state-of-the-art time division multiple access (TDMA). Finally, end-to-end simulations validate the system performance.

Index Terms-Satellite communications, spectral and power efficiencies, synchronization, OFDMA, SC-FDMA, FBMC.
\end{abstract}

\section{INTRODUCTION}

In order to serve the increasing demand for satellite broadband [1], next generation HTS systems need to offer higher system throughput and user data rates, flexibility to adapt to traffic demand across the coverage area, and at the same time decrease the cost per transmitted bit. Waveforms such as OFDMA and SC-FDMA are at the heart of terrestrial mobile long-term evolution (LTE) networks due to their high spectral efficiency and flexible traffic allocation. In addition, FBMC is one of the candidates for 5 -th generation (5G) networks [2].

In this paper, we present key solutions addressing the synchronization and non-linear distortion challenges for the application of these waveforms in the return link of GEO HTS systems. A synchronization acquisition approach based in GPS information is proposed. Several algorithms for timing and frequency offset estimation and synchronization tracking have been compared against the Cramer-Rao bound (CRB) and in terms of complexity in $[3,4]$. In this paper, the most

This paper has been accepted for publication in the Proceedings of the European Conference on Networks and Communications 2015 (EUCNC 2015), 29 June - 02 July 2015, Paris, France, DOI: 10.1109/EuCNC.2015.7194030.

(C) 2015 IEEE. Personal use of this material is permitted. Permission from IEEE must be obtained for all other uses, in any current or future media, including reprinting/republishing this material for advertising or promotional purposes, creating new collective works, for resale or redistribution to servers or lists, or reuse of any copyrighted component of this work in other works. promising algorithms are implemented in a practical satellite communication chain, including a realistic Ka band solid state power amplifier (SSPA) model at the user terminal, carrier frequency offset (CFO) and timing error (TE). Significant improvements of the detector performance are presented even at a low signal-to-noise ratio (SNR). In addition, an IMI cancelling receiver has been paired with static data pre-distortion at the transmitter [4] and employed not only for OFDMA, but also for SC-FDMA and TDMA. The power efficiency is maximized by means of output back-off (OBO) optimization and total degradation (TD) minimization. The overall spectral efficiency is also compared. Improvements are reported when employing guard subcarriers to protect from CFO for FBMC. Finally, endto-end simulations are performed to validate the performance of the system.

The rest of the paper is organized as follows. Section II presents the system model. Section III elaborates on the synchronization solutions, while Section IV presents the comparison and improvements of the spectral and power efficiencies. Finally, Section V concludes the paper.

\section{System MOdel}

In a digital transmission scheme [4], $N$ information symbols $s$ are used to modulate a signal $x$, which is pulse shaped by means of a square root raised cosine filter (SRRCF) and passed through a digital-to-analog converter (DAC). The signal is upconverted for radio frequency (RF) transmission, e.g. in Ka band. Satellite channel impairments include linear and nonlinear distortions plus additive white Gaussian noise (AWGN). The received signal is down-converted and passed through an analog-to-digital converter (ADC) and a matched filter. After time, frequency and phase synchronization, the received signal, $y$, is demodulated and decoded to obtain the received bits.

\section{A. OFDMA}

In OFDMA, the signal bandwidth is split in $N$ orthogonal subcarriers, resulting in a high spectral efficiency [5]. The inverse fast Fourier transform (IFFT) and the FFT are employed as multiplexing and demultiplexing techniques at the transmitter and the receiver, respectively. Due to the commonly applied cyclic prefix (CP), inter-symbol interference (ISI) and 
multiple access interference (MAI) are mitigated, reducing the equalization effort to a single-tap equalizer.

\section{B. SC-FDMA}

In SC-FDMA, the frequency domain subcarriers $S$ are precoded by means of a $N$-point DFT. After subcarrier mapping, a $M$-point IFFT $(M>N)$ is used for multiplexing, and a CP is inserted. The multiple access is realized by localized FDMA (LFDMA) or interleaved FDMA (IFDMA). Since the bandlimited IFDMA and LFDMA signals exhibit similar peak-toaverage-power ratio (PAPR) [6], we focus on LFDMA.

\section{C. $F B M C$}

FBMC uses a filter bank for the IFFT and a windowing function to reduce the sidelobes of the individual subcarriers. A subcarrier only has an influence on its direct neighbors [5]. In the first type of FBMC, e.g. filtered multitone (FMT), the $N$ subcarriers do not overlap. They are separated by a guard band and have a spectral efficiency comparable to TDMA. Here, complex-valued modulation, e.g. quadrature amplitude modulation (QAM), can be used. In the second type of FBMC, the subcarriers overlap, and only modulations with real-valued symbols can be used, e.g. offset QAM called staggered multitone (SMT). The resulting spectral efficiency is similar to OFDMA [5]. The in-phase and quadrature components have a time offset of half the symbol interval. An efficient implementation of the prototype filter is achieved by means of a poly phase network (PPN) [5].

\section{SYNCHRONIZATION}

Synchronization in FFT-based waveforms is a topic investigated severally in literature [7], and some approaches have been implemented for the LTE air interface [8]. In the return link, a number of adjacent subcarriers are assigned to a user. The waveforms depend strongly on frame timing and carrier frequency synchronization of all users in order to mitigate ISI, inter-carrier interference (ICI) and MAI.

\section{A. Acquisition}

Synchronization acquisition focuses mainly on frame timing at $\log$-on to correct for differential propagation delays in the presence of residual frequency offsets and amplifier nonlinearity. Timing alignment of the uplink transmissions is achieved by applying a timing advance at each user terminal [8] to accommodate the differential propagation delays within the length of the CP. The physical random access channel (PRACH) in LTE is dimensioned with a guard time (GT) to accommodate the round trip time (RTT). The PRACH preamble is an SC-FDMA symbol derived from Zadoff-Chu (ZC) sequences due to their constant amplitude zero autocorrelation (CAZAC) properties $[9,10]$.

The requirements of synchronization acquisition of the FFTbased waveforms over a GEO satellite link are similar to those of LTE in terms of accuracy. However, the challenges are greater, mainly due to the larger propagation delays in the satellite channel with RTT of $500 \mathrm{~ms}$ as compared to an order of $\mu \mathrm{s}$ in the terrestrial link. Furthermore, the maximum

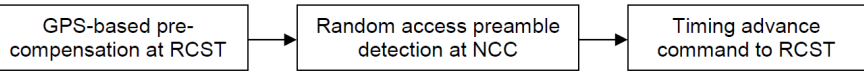

Fig. 1: GPS-based delay pre-compensation.

\begin{tabular}{|l|l|l|l|}
\hline Beam radius $(A)[\mathrm{km}]$ & 600 & 300 & 100 \\
\hline Speed of light $(B)[\mathrm{m} / \mathrm{s}]$ & \multicolumn{3}{|c|}{$3 * 10^{8}$} \\
\hline $\begin{array}{l}\text { Approx. max. 2-way differential } \\
\text { delay }(C=2 A / B)[\mathrm{ms}]\end{array}$ & 4 & 2 & 0.67 \\
\hline $\begin{array}{l}\text { Min. position accuracy of GPS } \\
\text { device }(D)[\mathrm{m}]\end{array}$ & \multicolumn{3}{|c|}{10} \\
\hline $\begin{array}{l}\text { Pre-compensation accuracy } \\
(E=2 D / B)[\mathrm{ns}]\end{array}$ & \multicolumn{3}{|c|}{67} \\
\hline
\end{tabular}

TABLE I: Pre-compensation accuracy.

\begin{tabular}{|l|l|l|l|}
\hline Carrier bandwidth $(A)[\mathrm{MHz}]$ & 100 & 150 & 250 \\
\hline $\begin{array}{l}\text { Subcarrier spacing for } N_{1}=1024 \\
\left(B=A / N_{1}\right)[\mathrm{KHz}]\end{array}$ & 97.7 & 146.5 & 244.1 \\
\hline $\begin{array}{l}\text { Symbol duration for } N_{1}=1024 \\
(C=1 / B)[\mu \mathrm{s}]\end{array}$ & 10.2 & 6.8 & 4.1 \\
\hline $\begin{array}{l}\text { Subcarrier spacing for } N_{2}=4096 \\
\left(D=A / N_{2}\right)[\mathrm{KHz}]\end{array}$ & 24.4 & 36.6 & 61 \\
\hline $\begin{array}{l}\text { Symbol duration for } N_{2}=4096 \\
(E=1 / D)[\mu \mathrm{s}]\end{array}$ & 40.8 & 27.2 & 16.4 \\
\hline
\end{tabular}

TABLE II: Symbol duration.

differential propagation delay within a GEO spot beam is of the order of ms. Given a typical OFDM symbol duration of the order of $\mu \mathrm{s}$, a guard time greater than the satellite RTT or even the maximum differential propagation delay is not affordable within an integrated PRACH. In similar fashion, the CP cannot be dimensioned for using cyclic shifts of a $\mathrm{ZC}$ sequence.

In order to solve these problems, we propose the use of GPS-based timing pre-compensation as shown in Fig. 1. A GPS device at each return channel via satellite terminal (RCST) can track its location. This can be combined with existing satellite and network control center (NCC) data to calculate the two-way propagation delay and pre-compensate it before transmitting the random access (RA) preamble. In this way, the timing misalignment between RCSTs can be reduced from ms to ns as shown in TABLE I. Given the relevant carrier bandwidths and FFT sizes [4], TABLE II shows that very low $\mathrm{CP}$ overhead for the symbol duration is required.

\section{B. Tracking and estimation}

After synchronization is acquired, estimation of residual timing and carrier frequency offsets is used for fine synchronization and tracking. The Mengali and Morelli (M\&M) algorithm [11] is chosen for tracking timing offset since it: 1) does not show a definite threshold effect in terms of SNR; 2) requires low complexity due to the adoption of two implementation strategies, one for small number of points and the other for larger sets; 3) has an estimation range independent from the number of points in the observed sequence. The Moose algorithm [12] is selected for tracking frequency offsets due to its closeness to the CRB. The remaining static phase offset 


\begin{tabular}{|c|c|}
\hline System FFT size & 128 carriers \\
\hline User FFT size & 16 subcarriers \\
\hline Cyclic prefix size & 1/8th system FFT size \\
\hline User pilot period & 10 SC-FDMA symbols \\
\hline
\end{tabular}

TABLE III: SC-FDMA simulation setup.

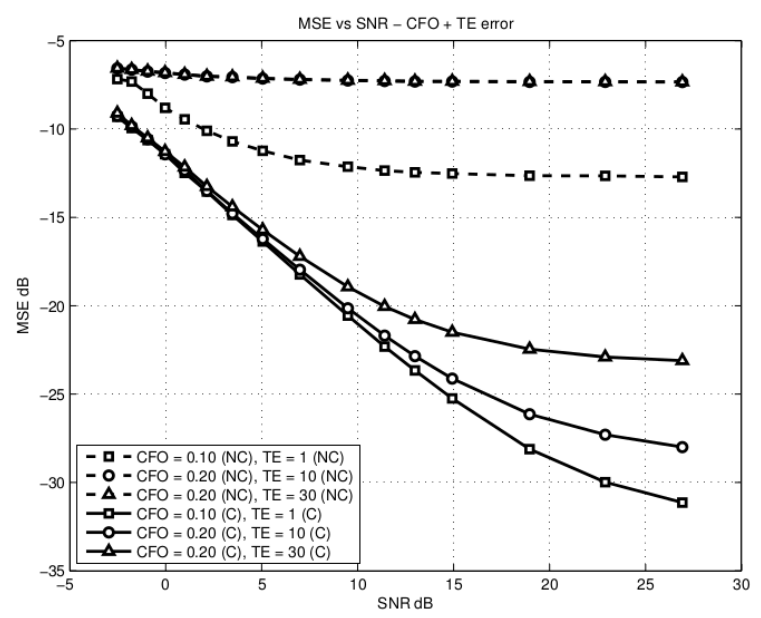

Fig. 2: MSE vs. SNR, CFO and TE compensation. NC stands for "Not Compensated", while C denotes "Compensated".

can be recovered using phase locked loop (PLL) filters before the signal is passed to the demodulator [3].

The algorithms are tested in the SC-FDMA setup in TABLE III. Fig. 2 shows the performance in terms of mean squared error (MSE) of the received constellation symbols at the input of the demodulator for different values of normalized CFO, TE and SNR. A normalized CFO of 0.1 means that terminal and gateway oscillators are misaligned by $0.1 * \Delta f$, where $\Delta f$ is the subcarrier spacing. TE of 1 means that the acquisition stage outputs a signal with a relative drift of 1 sample w.r.t. the nominal start of frame. The M\&M algorithm presents sufficiently low MSE at low SNR below $0 \mathrm{~dB}$, and the performance is degraded only when the TE approaches the size of the CP. The Moose algorithm also performs well at low SNR below $0 \mathrm{~dB}$. It is shown that the mutual cross-influence of CFO over timing offset estimation, or TE over frequency offset estimation, slightly degrades the overall receiver performance.

\section{SPECTRAL AND POWER EFFICIENCIES}

The power efficiency of the transmission schemes is maximized by means of an IMI cancelling receiver, optimization of $\mathrm{OBO}$ and minimization of TD in the non-linear channel with a high-power amplifier (HPA). In this setup, FBMC is expected to perform similarly to OFDMA. However, when $\mathrm{CFO}$ is added and guard subcarriers inserted, FBMC is shown to improve the power and spectral efficiencies. Finally, endto-end simulations validate the performance in a channel with non-linear distortion and frequency/timing offset.

\section{A. Power efficiency optimization}

Communication over the satellite channel suffers from nonlinear distortion due to the imperfect amplitude and phase

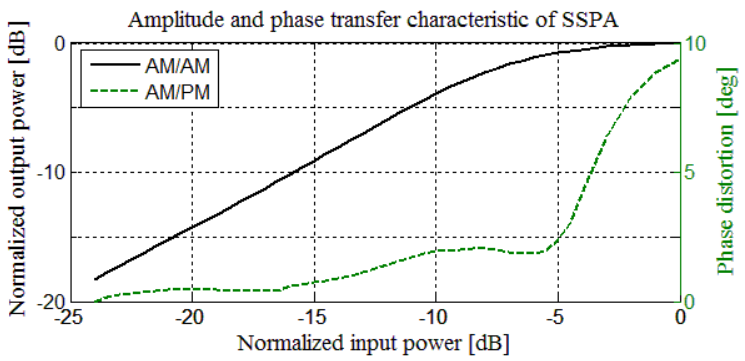

Fig. 3: Transfer characteristics of a Ka-band SSPA.

transfer characteristics of HPAs. In this study, the SNR requirement and spectral efficiency are compared for SC-FDMA, OFDMA and state-of-the-art TDMA in the return link. The measured characteristics of a physical Ka-band SSPA in the user terminal are presented in Fig. 3. In the DVB-RCS2 [13], QPSK, 8-PSK and 16-QAM are employed. Roll-off factors of $5 \%$ and $20 \%$ are applied with TDMA. In OFDMA and SC-FDMA, 2048 digital subcarriers per physical carrier are allocated to 32 users with 64 subcarriers per user. In addition, a $5 \%$ guard band is included between the physical carriers [8].

In state-of-the-art systems, signal detection is performed in the presence of the interfering component and the AWGN. However, the receiver can extract the useful signal information in the interfering component by means of IMI cancellation. It has been proposed in [14] for systems with OFDMA. However, if static data pre-distortion [4] is applied at the transmitter, the constellation warping at the receiver in systems like SCFDMA and TDMA is undone, i.e. the received centroids coincide with the original constellation. As a result, the IMI cancelling receiver can be applied also with SC-FDMA and TDMA. Here, a set of received symbols, e.g. a received frame, is buffered. In the first iteration of IMI cancellation, the symbols are demodulated. Based on the knowledge of the HPA transfer function, operating point (applicable with fixed mode or uplink power control) and the processing steps along the chain, an estimate of the received symbols can be obtained, which is subtracted from the buffered symbols to obtain an estimate of the interfering component. It is then subtracted from the buffered symbols to obtain a new set of received symbols, as an output of the first iteration of IMI cancellation. In the second iteration, the newly obtained received symbols are demodulated and used to better estimate the interfering component which is again subtracted from the originally buffered received symbols. The output of this process comprises of the newly obtained received symbols after a number of iterations. It is shown below that even two iterations can yield a significant gain. Finally, the buffer is released, and a new set of received symbols is processed.

In order to quantify the power efficiency penalty induced by the amplifier nonlinearity, the TD metric is defined as follows:

$$
\mathrm{TD}[\mathrm{dB}]=\mathrm{OBO}+\mathrm{SNR}_{\substack{\text { non-linear } \\ \text { channel }}}-\mathrm{SNR}_{\substack{\text { linear } \\ \text { channel }}} .
$$

The results are presented in Fig. 4. The chosen reference SNR targets in the linear channel are $3.7 \mathrm{~dB}, 8.9 \mathrm{~dB}$ and $10.5 \mathrm{~dB}$, respectively. These correspond to a bit-error ratio (BER) target 
TD vs. OBO of TDMA, SC-FDMA and OFDMA in AWGN with SSPA in RTN link, $E_{s} / N_{0}=3.7 \mathrm{~dB}$

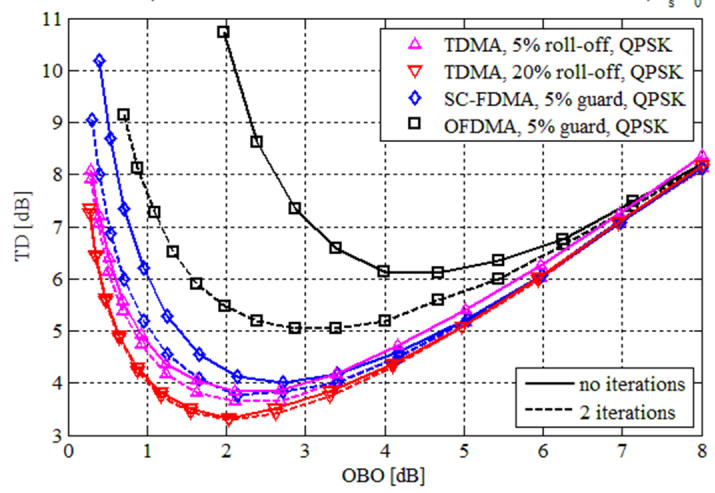

(a) TD of QPSK.

TD vs. OBO of TDMA, SC-FDMA and OFDMA in AWGN with SSPA in RTN link, $E_{\mathrm{s}} / N_{0}=10.5 \mathrm{~dB}$

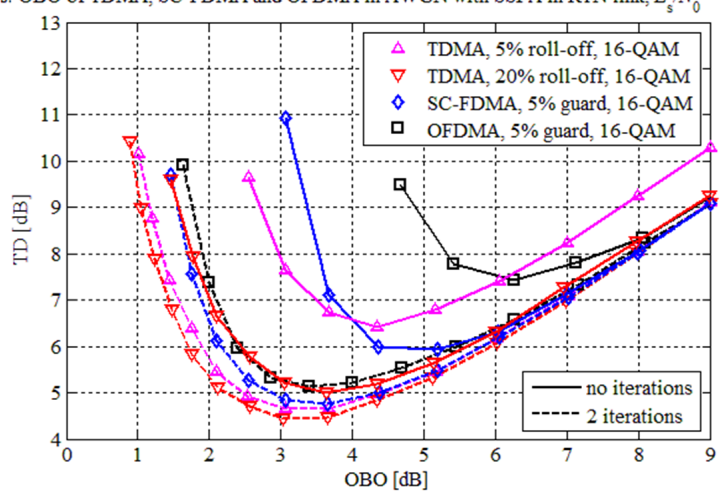

(c) TD of 16-QAM.

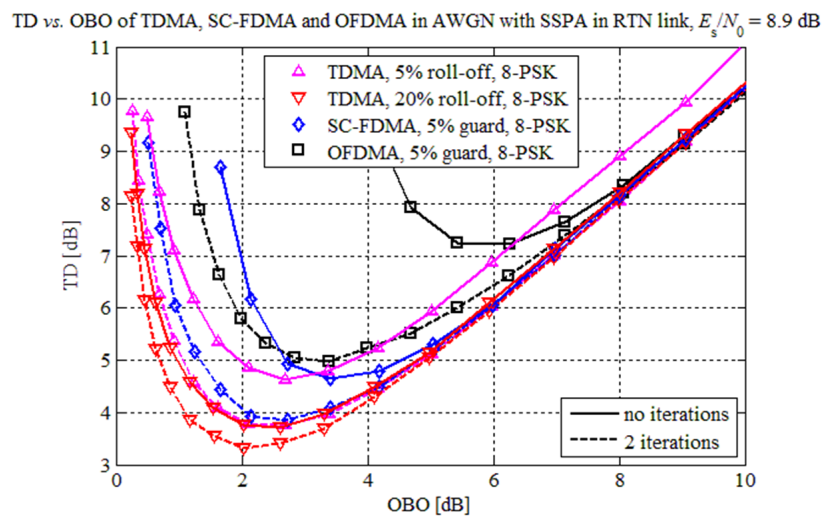

(b) TD of 8-PSK.

SE vs. $E_{\mathrm{s}} / N_{0}$ of TDMA, SC-FDMA and OFDMA in AWGN with SSPA in RTN link

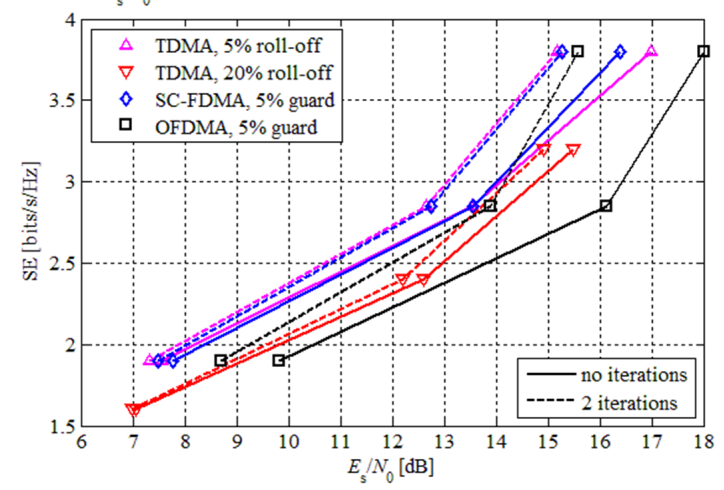

(d) Spectral and power efficiencies.

Fig. 4: Spectral and power spectral efficiencies of TDMA, OFDMA and SC-FDMA in return link.

of $10^{-3}$ to allow for forward error correction coding (FEC). Two iterations of IMI cancellation shift the minimum TD values to lower OBO values. For example, the optimum OBO for $16-\mathrm{QAM}$ is reduced from $3.6 \mathrm{~dB}$ to $2.95 \mathrm{~dB}$ in TDMA with $20 \%$ roll-off, from $4.35 \mathrm{~dB}$ to $3 \mathrm{~dB}$ in TDMA with $5 \%$ roll-off, from $5.17 \mathrm{~dB}$ to $3.63 \mathrm{~dB}$ in SC-FDMA and from 6.25 $\mathrm{dB}$ to $3.39 \mathrm{~dB}$ in OFDMA. The gains in power efficiency for the three modulation orders can be summarized as follows: $0.1-0.7 \mathrm{~dB}$ for TDMA with $20 \%$ roll-off, $0.2-1.8 \mathrm{~dB}$ for TDMA with $5 \%$ roll-off, $0.3-1.1 \mathrm{~dB}$ for SC-FDMA and $1.1-2.5 \mathrm{~dB}$ for OFDMA. Adding the minimum TD values to the SNR targets and considering the roll-off factors and the guard bands, the spectral efficiency is also shown in Fig. 4 as a function of the SNR requirement. SC-FDMA demonstrates an overall improvement of the power efficiency of more than 2 $\mathrm{dB}$ as compared to state-of-the-art TDMA with $20 \%$ roll-off.

\section{B. Spectral efficiency improvement}

In the return link, the received signal is the sum of all user contributions, each one with its own synchronisation offsets and non-linear distortion. These impairments can be mitigated with the insertion of guard band (GB) between users. We consider a scenario with three users, and we assume that the receiver is perfectly synchronized with the central user, whereas the others suffer CFO. Fig. 5 shows the BER of the outmost subcarriers of the central user, where most of the

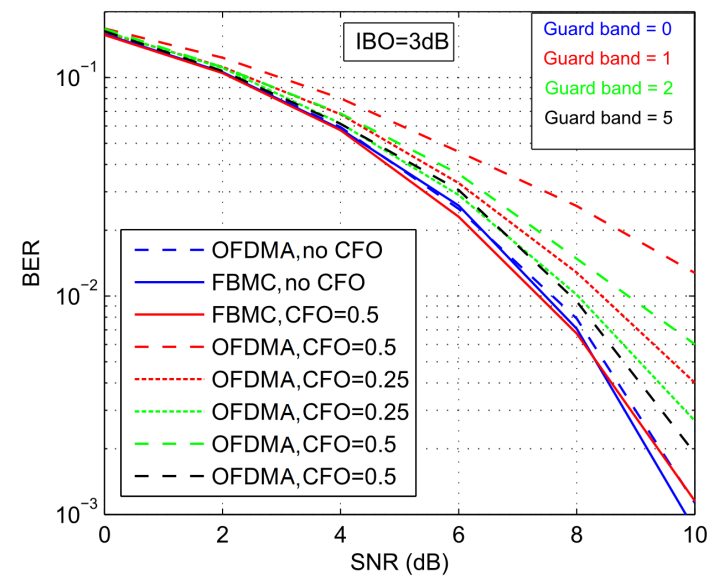

Fig. 5: BER of multi-user FBMC and OFDMA with QPSK for different guard band and CFO of adjacent users.

interference is present, with IBO $=3 \mathrm{~dB}$ and different CFO. If a single subcarrier between users is switched off as GB in FBMC, it outperforms OFDMA, even if the latter uses a higher number of GB subcarriers.

To better understand the spectral efficiency improvement of FBMC, we assume a multi-carrier system with 2048 subcarriers, where a single resource block (RB) of 12 subcarriers is assigned to a user. In OFDMA, $5 \%$ of GB between physical carriers is included. In FBMC, $2 \%$ of GB is considered to take into account non-linear distortions. TABLE IV shows the 


\begin{tabular}{|lcccccc|}
\hline & GB & GB & $N_{\mathrm{u}}$ & Eff. & $N_{\mathrm{u}}$ & Eff. \\
& carriers & users & $1 \mathrm{RB}$ & $1 \mathrm{RB}$ & $10 \mathrm{RB}$ & $10 \mathrm{RB}$ \\
\hline OFDMA & $5 \%$ & $0 \mathrm{SC}$ & 162 & $95 \%$ & 16 & $95 \%$ \\
OFDMA & $5 \%$ & $1 \mathrm{SC}$ & 149 & $87 \%$ & 16 & $94 \%$ \\
OFDMA & $5 \%$ & $2 \mathrm{SC}$ & 139 & $81 \%$ & 15 & $93 \%$ \\
SMT & $2 \%$ & $1 \mathrm{SC}$ & 154 & $91 \%$ & 16 & $97 \%$ \\
FMT & $2 \%$ & $0 \mathrm{SC}$ & 167 & $78 \%$ & 16 & $78 \%$ \\
\hline
\end{tabular}

TABLE IV: Comparing efficiencies of FBMC and OMT.

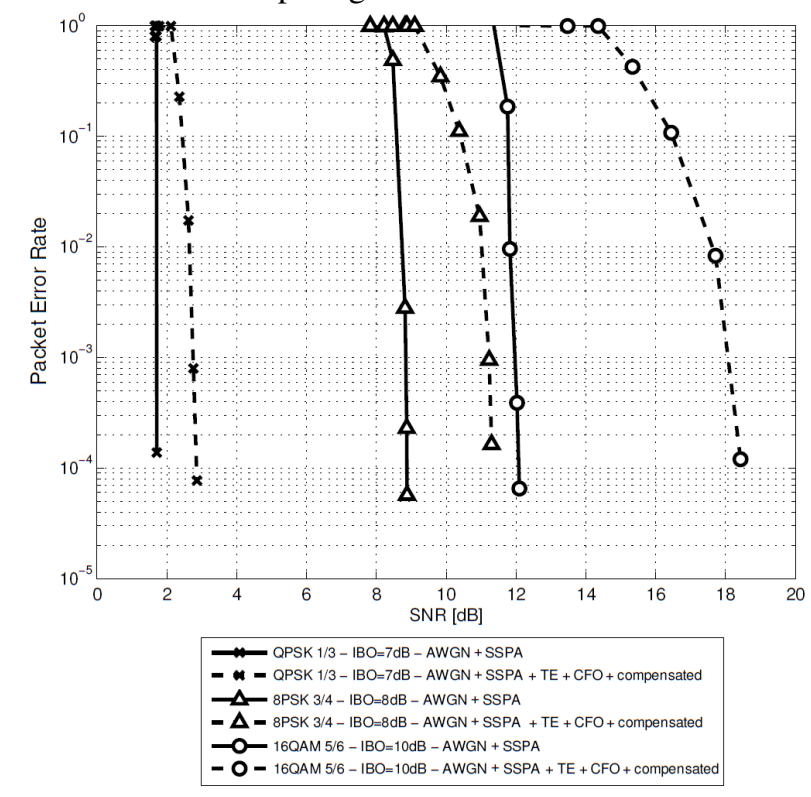

Fig. 6: End-to-end PER results.

efficiency in terms of percentage of subcarriers used for data over the total number of subcarriers. With $1 \mathrm{RB}$ assigned to each user, the best efficiency is achieved by OFDMA without any GB between users. However, as soon as the number of GB subcarriers is increased to reduce the effect of synchronization errors, SMT turns out to be more efficient than OFDMA. SMT efficiency increases, when a larger number of RBs is allocated to each user as reported in the table for 10 RBs. FMT has lower efficiency due to the non-overlapping filters, which allow avoiding GB between users but reduce the bandwidth utilization. Nevertheless FMT is even more robust than SMT to $\mathrm{CFO}$ and phase noise, and it can further relax the receiver complexity in asynchronous scenarios.

\section{End-to-end performance consolidation}

The system performance has been validated in an end-toend return link SC-FDMA simulator with DVB-RCS2 FEC. The packet-error rate (PER) is presented in Fig. 6 for CFO = 0.1 and $\mathrm{TE}=5$. The performance curves have been obtained by setting the OBO of the SSPA according to the minimum TD values without IMI cancellation from Fig. 4. Increasing the modulation order, the PER results show an increasing gap between a reference scenario only with SSPA and AWGN and the scenario, where TE and CFO are compensated with the M\&M and Moose algorithms. This is due to the higher sensitivity of higher order modulations to distortions in the channel. Using
Fig. 2, this behaviour can be explained by the fact that for low SNR the system is noise-limited, while at higher SNR, where 16-QAM operates, the system is limited by the residual offsets, which it is not able to completely compensate. Nonetheless, the the M\&M and the Moose algorithms are essential to enable quasi error-free performance of the higher order modulations albeit at an increased SNR requirement.

\section{CONClusion}

In this paper, the terrestrial mobile waveforms OFDMA, SCFDMA and FBMC have been evaluated for application over the return link GEO satellite channel. GPS-based delay precompensation has been shown to enable synchronization acquisition with negligible CP overhead. Timing and frequency offsets are best estimated and tracked by means of the M\&M algorithm and the Moose algorithm, respectively, showing significant MSE improvement even at low SNR. The power efficiency has been maximized by means of an IMI cancelling receiver. SC-FDMA demonstrated an overall power efficiency gain of more than $2 \mathrm{~dB}$ as compared to state-of-the-art TDMA. The proposed mechanisms to cope with the impairments of the satellite channel in terms of nonlinearity and synchronization challenges promote SC-FDMA as a promising alternative to TDMA. Due to its ability to cope with asynchronous access and flexible spectrum allocation, FBMC is also an appealing alternative for the satellite return link.

\section{ACKNOWLEDGEMENT}

This work has been supported by the BATS research project funded by the EU-FP7 under contract n 317533 .

\section{REFERENCES}

[1] Broadband Access via Integrated Terrestrial and Satellite Systems (BATS), "ICT-2011.1.1 BATS D4.1: Satellite Network Mission Requirements," European Project, Tech. Rep., 2012.

[2] 5th Generation Non-Orthogonal Waveforms for Asynchronous Signalling (5GNOW), "D3.1: 5G Waveform Candidate Selection," European Project, Tech. Rep., 2013.

[3] D. Benfatto, N. Privitera, R. Suffritti, A. Awoseyila, B. Evans, and S. Dimitrov, "On Acquisition and Tracking Methods for SC-FDMA over Satellite," in in Proc. of 7th Advanced Satellite Multimedia Systems Conference (ASMS2014), Livorno, Italy, Sep. 8-10 2014.

[4] S. Dimitrov, G. Boccolini, S. Jaeckel, D. Benfatto, N. Privitera, R. Suffritti, A. A., and B. Evans, "FFT-based Waveforms for High Through Satellite Communications: Opportunities and Challenges," in in Proc. of 20th Ka and Broadband Communications Conference, Vietri sul Mare Salerno, Italy, Oct. 1-3 2014

[5] B. Farhang-Boroujeny, "OFDM versus Filter Bank Multicarrier," IEEE Signal Processing Magazine, pp. 92-112, May 2011.

[6] H. Ochiai, "On Instantaneous Power Distributions of Single-carrier FDMA Signals," IEEE Wireless Communications Letters, vol. 1, no. 2, pp. 73-76, Apr. 2012

[7] M. Morelli, C.-C. J. Kuo, and M.-O. Pun, "Synchronization Techniques for Orthogonal Frequency Division Multiple Access (OFDMA): A Tutorial Review," Proceedings of the IEEE, vol. 95, no. 7, pp. 13941427, Jul. 2007.

[8] Technical Specification Group Radio Access Network; Evolved Universal Terrestrial Radio Access (EUTRA); Physical Layer Procedures (Rel. 11), 3rd Generation Partnership Project Std. 3GPP TS 36.213, 2013.

[9] D. Chu, "Polyphase Codes with Good Periodic Correlation Properties," IEEE Transactions on Information Theory, vol. 18, no. 4, pp. 531-532, Jul. 1972.

[10] Y. Wen, W. Huang, and Z. Zhang, "CAZAC sequence and its Application in LTE Random Access," in in Proc. of IEEE Information Theory Workshop, Oct. 2006, pp. 544-547. 
[11] U. Mengali and M. Morelli, "Data-aided Frequency Estimation for Burst Digital Transmission," IEEE Transactions on Communications, vol. 45, no. 1, pp. 23-25, Jan. 1997.

[12] P. H. Moose, "A Technique for Orthogonal Frequency Division MultMultiple Frequency Offset Correction," IEEE Transactions on Communications, vol. 42, no. 10, pp. 2908-2914, Oct. 1994.

[13] Second Generation DVB Interactive Satellite System (DVB-RCS2); Part 2: Lower Layers for Satellite Standard, Digital Video Broadcasting (DVB) Std. ETSI EN 301 545-2, Jan. 2012.

[14] H. Chen and A. M. Haimovich, "Iterative Estimation and Cancellation of Clipping Noise for OFDM Signals," IEEE Communications Letters, vol. 7, no. 7, pp. 305-307, Jul. 2003. 